\title{
SUBSTRATE AND ANNEALING INFLUENCES ON THE RESIDUAL STRESS OF POLYSILICON
}

\author{
Thomas W. Kim, Bishnu Gogoi, Ken G. Goldman, Andrew C. McNeil, Nicholas J. Rivette, \\ Stephen E. Garling, and Daniel J. Koch \\ Motorola, Semiconductor Products Sector, Transportation Systems Group, MEMS1 \\ Phoenix, AZ 85008
}

\begin{abstract}
Our study reports the compressive and tensile residual stresses of LPCVD undoped polysilicon (poly-Si) deposited over three different substrates-silicon oxide $\left(\mathrm{SiO}_{2}\right)$, densified phosphosilicate glass (PSG), and silicon nitride $\left(\mathrm{Si}_{3} \mathrm{~N}_{4}\right)$. Using the design of experiment methodology, the thickness of the polysilicon film was varied from $0.1 \mu \mathrm{m}$ to $3.2 \mu \mathrm{m}$ and the asdeposited stress values (as well as the residual stresses after annealing at four different conditions) were recorded. Stress profiles as a function of poly-Si thickness for each polysubstrate group and for each anneal condition were obtained. SEM images were also taken to reveal the texture of each polySi film surface, enabling a qualitative discussion of the different crystallographic states and the various mechanisms of island and void formation for poly-Si. Our results have direct engineering applications for MEMS surface micromachined devices by providing residual stress values of poly-Si in conjunction with widely used substrates such as stoichiometric silicon nitride, thermal oxide, and PSG, before and after annealing. It assists endeavors to create more accurate micromechanical models and to construct yet unrealized micromachined devices.
\end{abstract}

\section{INTRODUCTION}

Polysilicon (Poly-Si) is commonly used as a mechanical structure or a sacrificial layer in various surface micromachined MEMS applications [1]. Because the film is isotropic and has superior material properties compared to metal films, polysilicon is the structural material of choice when designing surface micromachined sensors and actuators [2]. Numerous deposition and processing techniques have been documented for both thin $(<2 \mu \mathrm{m}$ ) and thick (up to $10 \mu \mathrm{m}$ ) polysilicon $[3,4]$ but material characterization of thin film polysilicon (especially in the thickness range of $0.1 \mu \mathrm{m}$ to $1 \mu \mathrm{m}$ ) is still required. Oftentimes, thin film polysilicon is employed in devices where the parametric electrical behavior of the sensor is dependent on the film's mechanical properties. Also, polysilicon is used in conjunction with other MEMSapplication films such as thermal silicon oxide $\left(\mathrm{SiO}_{2}\right)$, densified phosphosilicate glass (PSG), and silicon nitride $\left(\mathrm{Si}_{3} \mathrm{~N}_{4}\right)$.

Deposited thin films can produce stress on a micromachined structure. In order to study the effects of poly on various substrates, radius of curvature, $R$, measurements were made using a wafer bowing system. Stress was calculated using Stoney's equation:

$$
\sigma=\frac{E_{s} t_{s}^{2}}{6\left(1-v_{s}\right) R t_{f}}
$$

where $E_{S} 1-v_{\mathrm{s}}$ is the biaxial modulus of the silicon substrate, $t_{s}$ is the thickness of the substrate, $t_{f}$ is the thickness of the deposited film, and $\sigma$ is the calculated film stress. The equation assumes that stress is equi-biaxial and homogeneous over the entire substrate.

Thin films have different material properties than their bulk material counterparts. Residual stresses of thin films often depend on deposition parameters. Polysilicon initially forms an amorphous solid that subsequently may crystallize during the deposition process. Highly columnar poly-Si films arise from a seed layer of random, small polysilicon grains formed by the bombardment of adatoms on the substrate. During thin film nucleation, intrinsic stresses caused by lattice mismatch, interstitial effects, and/or unequal growth mechanisms change the material properties of the thin film. Extrinsic stresses, such as non-uniform plastic deformation and thermal expansion (expressed quantitatively by the thermal expansion coefficient, CTE) may also change the behavior of the thin film.

In our study, the stress measurement of the resulting thin deposits are confounded with the type of substrate deposited beneath the polysilicon. Thermal $\mathrm{SiO}_{2}$ is readily employed as a sacrificial layer or dielectric film for passivation in surface micromachining. It is a compressive film. Stoichiometric LPCVD $\mathrm{Si}_{3} \mathrm{~N}_{4}$ is tensile and is used mainly as an isolation and buffer layer. Designers tend to shy away from using thermal oxide and/or nitride as mechanical components because of their high residual stress. Phosphosilicate glass (PSG) etches rapidly in $\mathrm{HF}$ and is used as a sacrificial layer. Due to its low melting temperature (approximately $950^{\circ} \mathrm{C}$ ), PSG softens and flows under standard anneal conditions. The residual stress profiles for poly-Si on PSG can be expected to behave differently than poly-Si on nitride or thermal oxide.

Annealing and doping are popular techniques employed in MEMS applications to reduce the residual compressive and tensile stresses of as-deposited polysilicon [5]. Not only does annealing reduce residual stresses caused by the differences in the thermal expansion coefficient of the film-substrate interface and the non-uniform growth of the film, it also provides a means to increase the surface roughness of the film preventing stiction/delamination of further deposited films. SEM images of the poly-Si film surface before anneal provides qualitative information on the rate of island and void formation.

\section{EXPERIMENTAL METHODS}

A screening experiment was run following a set design of experiment (DOE) (Table 1). Low thicknesses of poly-Si on LPCVD silicon nitride were not sought because rarely are the two combined with the given film thicknesses in sensor applications. A Tencor ${ }^{\mathrm{TM}}$ FLX-2320 system monitored the radius of curvature on $100 \mathrm{~mm}$ dia., p-type $<100\rangle$ silicon test wafers. Bare silicon test wafers were baselined after deposition of the three different substrates-nitride, oxide, and PSG. Each 
wafer was annealed for further stress analysis to $900^{\circ} \mathrm{C}(1 \mathrm{hr})$, $950^{\circ} \mathrm{C}(1 \mathrm{hr}), 1000^{\circ} \mathrm{C}(1 \mathrm{hr})$, or $1100^{\circ} \mathrm{C}$ (RTA, $\left.1 \mathrm{~min}\right)$.

Table 1. Design of Experiment. The table indicates the number of test wafers in each cell deposited to the set compressive (-) or tensile (+) poly-Si thickness with a corresponding substrate.

\begin{tabular}{|c|c|c|c|}
\hline Poly-Si & w/Si( $)_{2}$ & w/PSC & $\mathrm{w} / \mathrm{Si}_{3} \mathrm{~N}_{4}$ \\
\hline $0.1 \mu \mathrm{m}$ & 8 & 8 & -- \\
\hline $0.2 \mu \mathrm{m}$ & 8 & 8 & -- \\
\hline $0.4 \mu \mathrm{m}$ & 8 & 8 & -- \\
\hline $0.8 \mu \mathrm{m}$ & 8 & 8 & 8 \\
\hline $1.6 \mu \mathrm{m}$ & 8 & 8 & 8 \\
\hline $3.2 \mu \mathrm{m}$ & 8 & 8 & 8 \\
\hline
\end{tabular}

LPCVD undoped poly-Si was deposited using a vertical thermal reactor with silane $\left(\mathrm{SiH}_{4}\right)$ as the main gas component at approximately $600^{\circ} \mathrm{C}$. (Note that only the deposition time was altered to achieve greater thicknesses. All other deposition conditions were held constant.) With some adjustments in silane flow rate, pressure, and deposition temperature, both compressive and tensile poly-Si films were produced. A wet $\mathrm{KOH}$ bath system was used for subsequent backside etching. A Prometrix ${ }^{\text {TM }}$ FT-750 system with film specific algorithms was used to measure the thickness of all the films. Only one poly-Si film (in compression at $3.2 \mu \mathrm{m}$ ) could not be read due to the "hazy" quality of the film's refraction.

Standard procedures were used to deposit the three substrates. A dry oxidation process was employed to deposit $0.04 \mu \mathrm{m}$ of thermal oxide. Phosphite was used as the phosphorous dopant for $1 \mu \mathrm{m}$ of PSG and the glass was densified at $950^{\circ} \mathrm{C}$. A vertical LPCVD furnace was employed for $0.14 \mu \mathrm{m}$ thick stoichiometric nitride deposition with dichlorosilane (DCS) and ammonia $\left(\mathrm{NH}_{3}\right)$ as the main gaseous components. All anneals occurred in a horizontal furnace with nitrogen gas save the rapid thermal anneal process.

\section{RESULTS AND DISCUSSION}

Figure 1 shows the stress profile for compressive poly-Si films deposited over three different substrates. As the thickness of the poly-Si film increases, the absolute stress values decrease. The profiles for poly on nitride and poly on oxide are similar at thicknesses greater than $0.8 \mu \mathrm{m}$. Although at room temperature thermal oxide is compressive and nitride is tensile, very thin films were used as substrates in this experiment $(0.14 \mu \mathrm{m}$ for nitride and $0.04 \mu \mathrm{m}$ for oxide). At high poly film thicknesses, there is perhaps enough poly to mask the effect of the substrate on the film. The trend is linear at these greater thicknesses with the stress value approaching $200 \mathrm{MPa}$ at $3.2 \mu \mathrm{m}$. Nevertheless, a difference is perceived through the entire DOE space for poly on $1 \mu \mathrm{m}$ PSG. PSG tends to be "inelastic," becoming viscous at high temperatures. At low poly thicknesses, poly on PSG has higher compressive stress values than the other two film-substrate groups. In turn, at high poly-Si thicknesses, poly on PSG has lower compressive stress values. For low thicknesses of poly-Si, the higher stress values could be the result of more severe lattice mismatch between the polysilicon and PSG as well as misfit stresses caused by interstitial impurities.

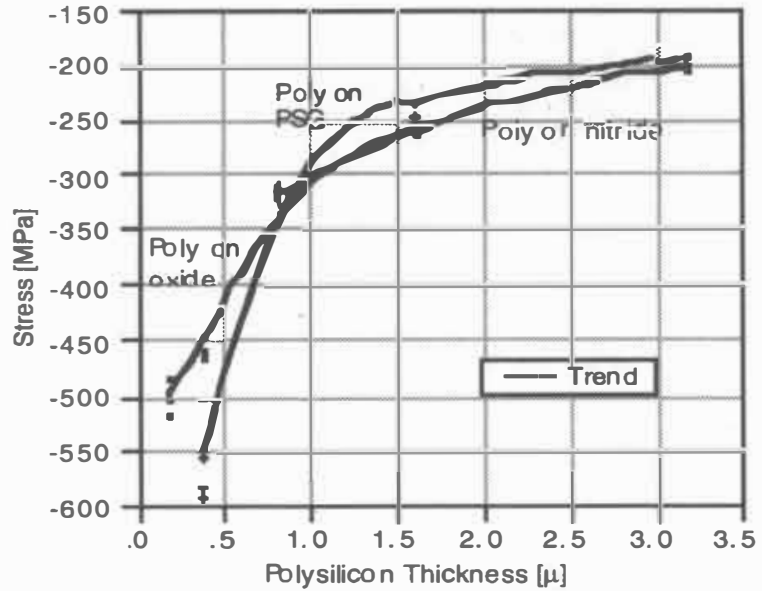

Figure 1. As-deposited compressive stress vs. poly-Si thickness for poly on three different substrates: $0.04 \mu \mathrm{m}$ thermal oxide, $0.14 \mu \mathrm{m}$ LPCVD nitride, and $1 \mu \mathrm{m}$ densified PSG.

Krulevitch has suggested that low thickness compressive polysilicon films are made up of small grains that are misoriented [6]. The adatoms do not have enough surface mobility to find the lowest energy levels; that is, the film is amorphous at first. As-deposited compressive stress values for very thin polysilicon films tend to be high as seen in Figure 1. As the deposition time is increased, however, polysilicon film thickness increases by favoring those grains preferentially oriented in the z-direction. Polysilicon films begin to grow vertically in a columnar fashion, and distinct islands arise changing the initially amorphous film into a crystalline structure. This competitive grain growth mechanism explains the crystallographic structure of compressive thin film polysilicon at higher thicknesses. As-deposited stresses decrease as poly-Si thickness increases. Figure $2 \mathrm{a}$ shows the evolution of polysilicon into a highly crystalline film. Note the rise of the columnar structure with islands approximately $0.75 \mu \mathrm{m}$ to $4.5 \mu \mathrm{m}$ across.

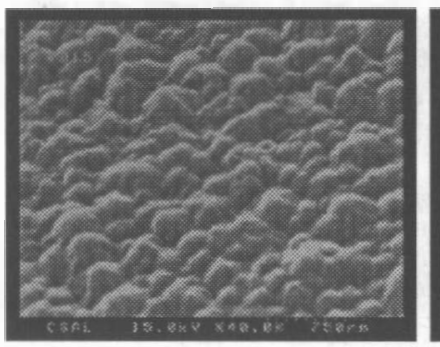
(a) 2. Pre-anneal SEM images of (a) $1.6 \mu \mathrm{m}$ compressive and (b) $1.3 \mu \mathrm{m}$ tensile poly-Si deposited on $0.04 \mu \mathrm{m}$ thermal oxide. Images were magnified $40 \mathrm{k}$ times and were taken at a $52^{\circ}$ tilt.

Tensile polysilicon films behaved differently. As poly-Si thickness increased to $2.5 \mu \mathrm{m}$, the as-deposited tensile stress values continued to increase, leveling off at approximately 275 to $300 \mathrm{MPa}$ (see Figure 3). As expected, poly on PSG had greater tensile stress values by about $40 \mathrm{MPa}$ throughout the DOE space and the profiles for poly on nitride and poly on thermal oxide are similar. There appears to be little dependence 
on poly-Si thickness for stress values at film thicknesses greater than $1.3 \mu \mathrm{m}$.

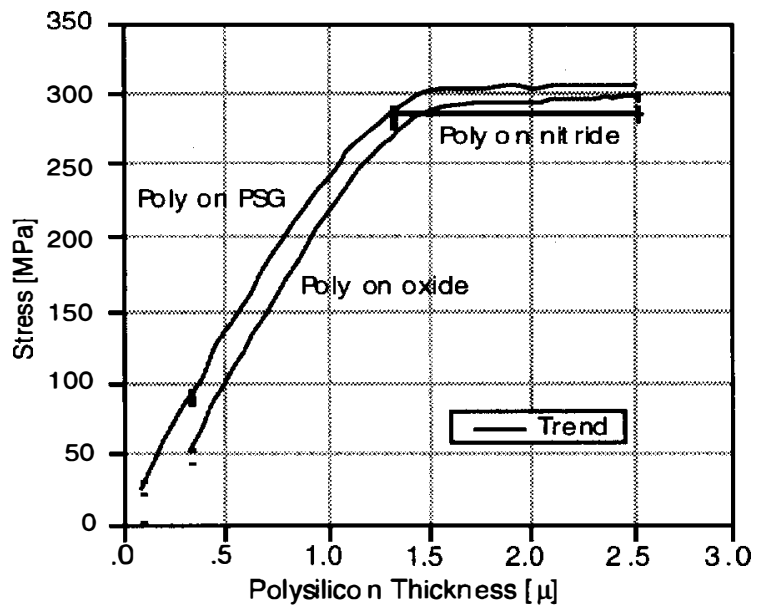

Figure 3. As-deposited tensile stress vs. poly-Si thickness for poly on three different substrates: $0.04 \mu \mathrm{m}$ thermal oxide, 0.14 $\mu m$ LPCVD nitride, and $1 \mu m$ densified PSG.

Tensile films are created when intermediate gases are driven off during deposition, leaving gaps within the film's microstructure. (Tensile films tend to contract.) Microvoids are expected since lateral diffusion of atoms evolves too slowly to fill all these gaps. A SEM image of tensile polysilicon revealed its amorphous structure (see Figure $2 b$ ). As poly-Si thickness increases, microvoids become more apparent. The "cobblestone" texture of $1.3 \mu \mathrm{m}$ thick poly was present for 0.4 $\mu \mathrm{m}$ and $2.5 \mu \mathrm{m}$ samples as well. The $1.3 \mu \mathrm{m}$ sample shows unequal vertical growth most likely due to interstitial impurities.

Thermal stresses are the highest contributors to the overall stress value. Annealing reduces stresses caused by thermal mismatch expansions and non-uniform nucleation of the film. For polysilicon, annealing at temperatures well above its deposition temperature (which is approx. $600^{\circ} \mathrm{C}$ ) should allow the atoms to find the lowest energy level, changing amorphous structures into crystalline forms. Figure 4 displays the residual stress profiles of compressive polysilicon (deposited on three different substrates) after being processed to four different anneal conditions. In general, as the anneal temperature increased, the film had lower compressive stress values. Also, the residual stress was independent of film thickness. (At film thicknesses less than approx. $1 \mu \mathrm{m}$ though, compressive stress values did have a dependence on poly-Si thickness. At higher thicknesses, however, it was mainly the temperature that drove the relaxation.) The rapid thermal anneal process (RTA) had promising results, bringing the residual compressive stress values for poly on oxide to less than $25 \mathrm{MPa}$ and to less than $50 \mathrm{MPa}$ for compressive poly on PSG. RTA did, however, push the compressive poly on nitride into the tensile regime, but this may be an indication of the set temperature [7]. A hot temperature excursion should be run with these samples to further understand the changes in thermal expansion.

Figure 5 shows residual stress plots of tensile polysilicon films (deposited on three different substrates) after annealing. Similar conclusions may be drawn about the effect of annealing on these tensile samples as with the compressive samples.
Temperature seems to drive the different levels of relaxation and there is little dependence on poly-Si thickness (above $1 \mu \mathrm{m}$ ). Again, higher temperatures produced lower residual stress films.
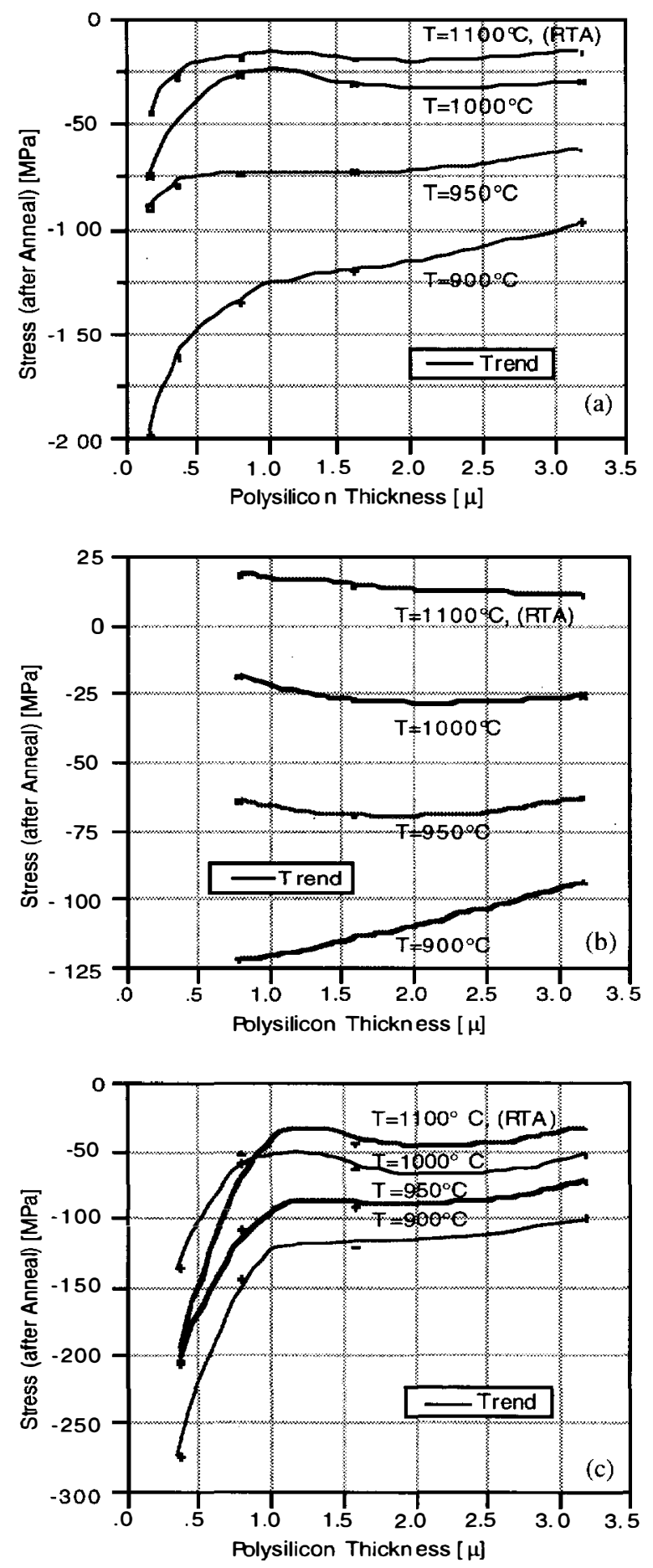

Figure 4. Residual stress profiles of compressive polysilicon as a function of the film's thickness. The poly-Si film is deposited on (a) $0.04 \mu \mathrm{m}$ thermal oxide, (b) $0.14 \mu \mathrm{m}$ LPCVD nitride, and (c) $1 \mu \mathrm{m}$ densified PSG. Samples at $900^{\circ} \mathrm{C}, 950^{\circ} \mathrm{C}$ and $1000^{\circ} \mathrm{C}$ were annealed for one hour while samples at $1100^{\circ} \mathrm{C}$ were annealed for one minute using a rapid thermal anneal (RTA) process. 

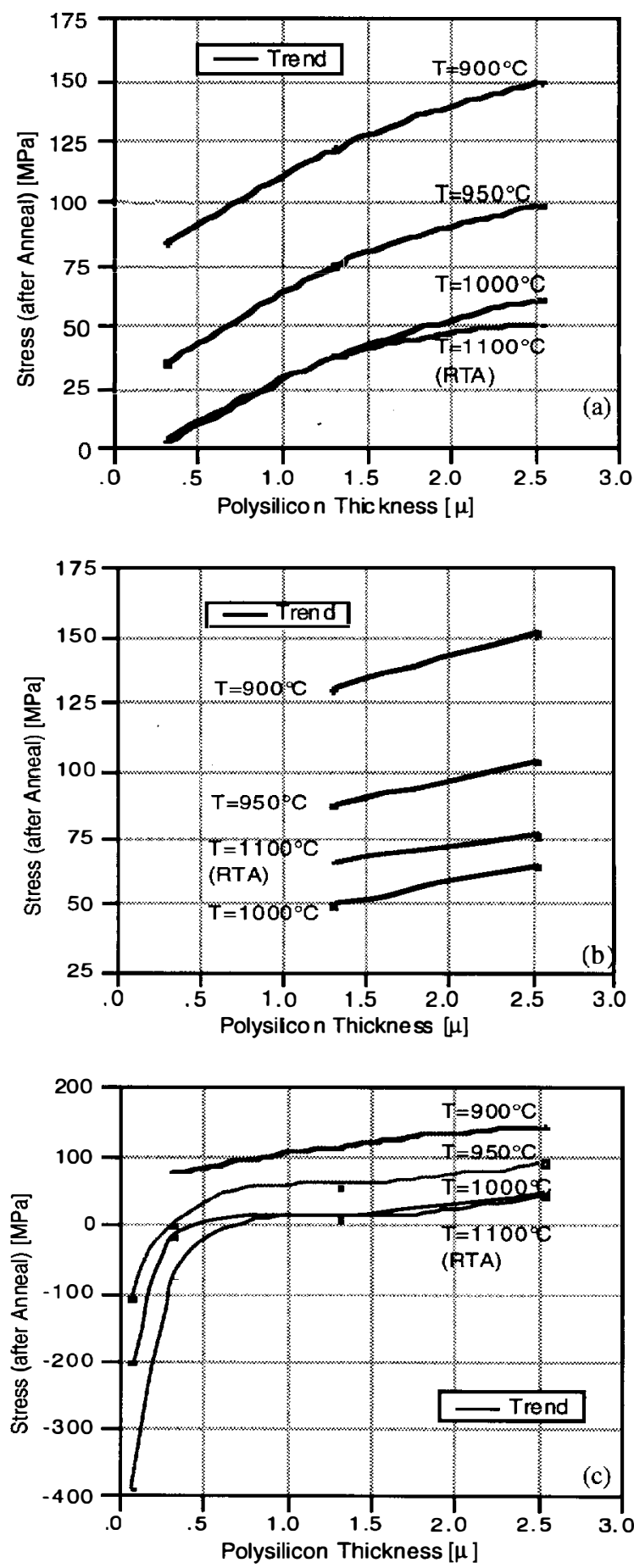

Figure 5. Residual stress profiles of tensile polysilicon as a function of the film's thickness. The poly-Si film is deposited on (a) $0.04 \mu \mathrm{m}$ thermal oxide, (b) $0.14 \mu \mathrm{m}$ LPCVD nitride, and (c) $1 \mu \mathrm{m}$ densified PSG. Samples at $900^{\circ} \mathrm{C}, 950^{\circ} \mathrm{C}$ and $1000^{\circ} \mathrm{C}$ were annealed for one hour while samples at $1100^{\circ} \mathrm{C}$ were annealed for one minute using a rapid thermal anneal (RTA) process.

\section{SUMMARY AND CONCLUSIONS}

Using the design of experiment (DOE) methodology, we examined compressive and tensile LPCVD polysilicon films (of
0.1 to $3.2 \mu \mathrm{m}$ thickness) deposited over three different substrates-silicon oxide $\left(\mathrm{SiO}_{2}\right)$, densified phosphosilicate glass (PSG), and silicon nitride $\left(\mathrm{Si}_{3} \mathrm{~N}_{4}\right)$. Stress measurements were made of as-deposited polysilicon films and stress profiles are provided for each poly-substrate group. Compressive polysilicon films had stress values ranging from 600 to 200 $\mathrm{MPa}$ as poly thickness increased from 0.1 to $3.2 \mu \mathrm{m}$. The profiles of stress vs. poly thickness for each different substrate revealed that poly on PSG has the lowest (most negative) stress values and that poly on oxide and poly on nitride have similar stress profiles. Tensile polysilicon films had stress values ranging from 5 to $300 \mathrm{MPa}$ as poly film thickness increased from 0.1 to $2.5 \mu \mathrm{m}$. Poly on PSG had higher stress values than poly on nitride and poly on oxide (indicating the "inelastic" nature of PSG).

Characterization of LPCVD polysilicon also included determining the stress of the film after four different anneal procedures: $900^{\circ} \mathrm{C}$ for $60 \mathrm{~min} ; 950^{\circ} \mathrm{C}$ for $60 \mathrm{~min} ; 1000^{\circ} \mathrm{C}$ for 60 $\mathrm{min}$; and $1100^{\circ} \mathrm{C}$ for $1 \mathrm{~min}$ (rapid thermal anneal, RTA). Generally, as the set anneal temperature increased, both compressive and tensile stresses approached 0 , independent of film thickness. This study contributes to the growing material analysis of polysilicon thin films and it provides new stress profiles (before and after anneal) of polysilicon used in MEMS manufacturing (i.e. in conjunction with nitride, oxide, or PSG).

\section{REFERENCES}

1. R. T. Howe, "Applications of Polysilicon Films in Microsensors and Microactuators", Materials Research Society Symposium Proceedings, 106, 213 (1988).

2. M. J. Madou, Fundamentals of Microfabrication, CRC Press, Boca Raton, 1997.

3. T. I. Kamins, Polycrystalline Silicon for Integrated Circuit Applications, Kluwer Academic Publishers, Boston, 1988.

4. M. Kirsten, B. Wenk, F. Ericson, J. A. Schweitz, W. Riethmüller, and P. Lange, "Deposition of Thick Doped Polysilicon Films with Low Stress in an Epitaxial Reactor for Surface Micromachining Applications," Thin Solid Films, 259, 181 (1995).

5. L. Ristic, M. L. Kniffin, R. Gutteridge, and H. G. Hughes, "Properties of Polysilicon Films Annealed by a Rapid Thermal Expansion Annealing Process," Thin Solid Films, 220, 106 (1992).

6. P. Krulevitch, G. C. Johnson, and R. T. Howe, "Stress and Microstructure in Phosphorous Doped Polycrystalline Silicon", Materials Research Society Symposium Proceedings, 276, 79 (1992).

7. H. Guckel, D. W. Burns, C. C. G. Visser, H. A. C. Tilmans, and D. Deroo, "Fine-Grained Polysilicon Films with Built-In Tensile Strain", IEEE Transactions on Electronic Devices, 35, 800 (1988). 